\title{
Prevalência de fatores de risco e proteção para doenças crônicas não transmissíveis em adultos: estudo transversal, Brasil 2012
}

doi: 10.5123/S1679-49742014000400003

\section{Prevalence of risk and protective factors for chronic diseases in adult population: cross-sectional study, Brazil 2012}

\author{
Deborah Carvalho Malta \\ Secretaria de Vigilância em Saúde, Ministério da Saúde, Brasília-DF, Brasil \\ Regina Tomie Ivata Bernal \\ Faculdade de Saúde Pública, Universidade de São Paulo, São Paulo-SP, Brasil \\ Marília Lavocat Nunes \\ Secretaria de Vigilância em Saúde, Ministério da Saúde, Brasília-DF, Brasil \\ Max Moura de Oliveira \\ Secretaria de Vigilância em Saúde, Ministério da Saúde, Brasília-DF, Brasil \\ Betine Pinto Moehlecke Iser \\ Secretaria de Vigilância em Saúde, Ministério da Saúde, Brasília-DF, Brasil \\ Faculdade de Medicina, Universidade do Sul de Santa Catarina, Tubarão-SC, Brasil \\ Silvânia Suely Caribé de Araújo Andrade \\ Secretaria de Vigilância em Saúde, Ministério da Saúde, Brasília-DF, Brasil \\ Rafael Moreira Claro \\ Faculdade de Enfermagem, Universidade Federal de Minas Gerais, Belo Horizonte-MG, Brasil \\ Carlos Augusto Monteiro \\ Núcleo de Pesquisas Epidemiológicas em Nutrição e Saúde, Universidade de São Paulo, São Paulo-SP, Brasil \\ Jarbas Barbosa da Silva Jr \\ Secretaria de Vigilância em Saúde, Ministério da Saúde, Brasília-DF, Brasil
}

Grupo de elaboração do Vigitel 2012

\section{Resumo}

Objetivo: descrever as prevalências de fatores de risco e proteção para doenças crônicas na população adulta brasileira e analisar as diferenças segundo variáveis sociodemográficas. Métodos: estudo transversal com dados de 2012 do Sistema de Vigilância de Fatores de Risco e Proteção para Doenças Crônicas por Inquérito Telefônico (Vigitel), incluindo residentes nas capitais brasileiras com idade $\geq 18$ anos; foram calculadas as prevalências e respectivos intervalos de confiança de $95 \%$ $\left(\mathrm{IC}_{95 \%}\right)$ segundo sexo, faixa etária e escolaridade. Resultados: entre 45.448 entrevistados, a prevalência de tabagismo foi de $12,1 \%\left(\mathrm{IC}_{95 \%}: 11,5 \%-12,8 \%\right.$ ), inatividade física, 14,9\% ( $\left.\mathrm{IC}_{95 \%}: 14,2 \%-15,5 \%\right)$, consumo abusivo de bebidas alcoólicas, 18,4\% $\left(\mathrm{IC}_{95 \%}: 17,7 \%-19,2 \%\right)$, excesso de peso, $51,0 \%$ ( $\left.\mathrm{IC}_{95 \%}: 50,1 \%-51,9 \%\right)$ e obesidade, $17,4 \%\left(\mathrm{IC}_{95 \%}: 16,7 \%-18,1 \%\right)$; os fatores de risco estudados estiveram associados a maior idade, sexo masculino e menor escolaridade. Conclusão: a prevalência dos fatores de risco e proteção apresenta diferenças segundo características sociodemográficas, a serem consideradas nas estratégias de promoção da saúde.

Palavras-chave: Fatores de Risco; Doença Crônica; Inquéritos Epidemiológicos; Vigilância Epidemiológica; Estudos Transversais.

\begin{abstract}
Objective: to describe chronic disease risk and protective factor prevalence in the Brazilian adult population and analyze the differences according to socio-demographic variables. Methods: this was a cross-sectional study using data from the 2012 Surveillance System for Risk and Protective Factors (Vigitel) containing information about adults living in Brazilian state capitals. Prevalence and respective 95\% Confidence Intervals were estimated according to gender, age and education level. Results: 45,448 adults were interviewed. Prevalence was as follows: smoking 12.1\% (95\%CI: 11.5\%12.8\%); physical inactivity 14.9\% (95\%CI: 14.2\%-15.5\%); abusive alcobol consumption 18.4\% (95\% CI: 17.75\%-19.2\%); excess weight 51.0\% (95\%CI: 50.1\%-51.9\%) and obesity 17.4\% (95\%CI: 16.7\%-18.1\%). The risk factors studied were associated with being older, being male and having less schooling. Conclusion: there are differences in prevalence of risk and protective factors according socio-demographic patterns and these variations must be considered in health promotion strategies.
\end{abstract}

Key words: Risk Factors; Chronic Disease; Health Surveys; Epidemiological Surveillance; Cross-Sectional Studies.

Endereço para correspondência:

Deborah Carvalho Malta - Ministério da Saúde, Secretaria de Vigilância em Saúde, Departamento de Vigilância de Doenças e Agravos Não Transmissíveis e Promoção da Saúde (DANTPS), SAF Sul, Trecho 2, Lotes 5 e 6, Bloco F, Torre 1, Edifício Premium, Térreo, sala 15, Brasília-DF, Brasil. CEP: 70070-600

E-mail: deborah.malta@saude.gov.br 


\section{Introdução}

As doenças crônicas não transmissíveis (DCNT) são responsáveis por $63 \%$ das causas de morte no mundo ${ }^{1}$ e em função da sua magnitude e importância, em 2011, foi aprovada a Declaração Política na Reunião de Alto Nível das Nações Unidas, quando se estabeleceu o compromisso dos países membros por seu enfrentamento. ${ }^{2}$

No Brasil, também predominam as DCNT como principais causas de morte, responsáveis por $72 \%$ dos óbitos, em grande parte resultantes de quatro grupos de doenças (cardiovasculares, cânceres, diabetes e respiratórias crônicas). ${ }^{3}$ Em 2011, no Plano de Ações Estratégicas para o Enfrentamento das Doenças Crônicas Não Transmissíveis no Brasil, de 2011 a 2022, foram incluídas ações para 0 enfrentamento dessas doenças e de seus principais fatores de risco modificáveis (tabagismo, alimentação inadequada, inatividade física, consumo abusivo de bebidas alcoólicas). 0 Plano ainda define ações para redução da carga de DCNT e seus fatores de risco ${ }^{4,5}$ As evidências apontam que as DCNT podem ser largamente prevenidas por meio de ações de promoção de saúde. ${ }^{1}$

Para o monitoramento continuo dos principais fatores de risco e proteção das DCNT, em 2006, foi iniciado o Sistema de Vigilância de Fatores de Risco e Proteção para Doenças Crônicas por Inquérito Telefônico (Vigitel), realizado anualmente em todas as capitais e no Distrito Federal. ${ }^{6}$

0 objetivo deste artigo é descrever as prevalências dos fatores de risco e proteção para doenças crônicas na população adulta das capitais brasileiras, segundo sexo, idade e escolaridade no ano de 2012.

\section{Métodos}

Foi realizado estudo transversal utilizando dados do Vigitel 2012. Este sistema utiliza amostras probabilísticas da população adulta ( $\geq 18$ anos de idade) residente nas capitais dos estados brasileiros e no Distrito Federal, a partir do cadastro das linhas de telefone fixo das cidades, disponibilizado pelas principais operadoras no país a cada ano. São sorteadas 5.000 linhas telefônicas de cada cidade, divididas em réplicas (ou subamostras) de 200 linhas cada, para identificação das linhas residenciais ativas. Após con- firmada a elegibilidade da linha, é realizada a seleção do morador a ser entrevistado. ${ }^{6,7}$

Em 2012, o questionário do Vigitel englobou 83 questões sobre os seguintes temas:

a) características demográficas e socioeconômicas dos indivíduos;

b) padrão de alimentação e de atividade física;

c) peso e altura referidos;

d) consumo de cigarro e de bebidas alcoólicas; e

e) autoavaliação do estado de saúde e morbidade referida.

Para o monitoramento continuo dos principais fatores de risco e proteção das DCNT, em 2006, foi iniciado o Sistema de Vigilância de Fatores de Risco e Proteção para Doenças Crônicas por Inquérito Telefônico (Vigitel), realizado anualmente em todas as capitais e no Distrito Federal.

A entrevista do Vigitel baseia-se no sistema de entrevistas telefônicas assistidas por computador, em que as perguntas são lidas na tela e as respostas registradas diretamente, em meio eletrônico, permitindo pulos automáticos de questões não válidas decorrentes das respostas anteriores. ${ }^{7}$

Foram estudados os seguintes indicadores de fatores de risco para DCNT, medidos em valores percentuais de:

- fumantes;

- ex-fumantes;

- fumantes com consumo de 20 ou mais cigarros por dia;

- fumantes passivos no domicílio (não fumantes que relatam haver pelo menos um dos moradores do seu domicílio com o costume de fumar dentro de casa);

- fumantes passivos no local de trabalho (não fumantes que relatam haver pelo menos uma pessoa com o costume de fumar em seu ambiente de trabalho);

- adultos com excesso de peso [índice de massa corporal (IMC) $\left.\geq 25 \mathrm{~kg} / \mathrm{m}^{2}\right]$ e com obesidade (IMC $\left.\geq 30 \mathrm{~kg} / \mathrm{m}^{2}\right)$

- consumo de carnes com excesso de gordura (hábito de consumir carnes com gordura visível e ou frango 
com pele, para aqueles que consomem 0 alimento pelo menos uma vez por semana);

- consumo de leite com teor integral de gordura (hábito de consumir leite com teor integral de gordura, para aqueles que tomam leite pelo menos uma vez por semana);

- consumo regular de refrigerante (hábito de consumir refrigerante ou suco artificial em cinco ou mais dias por semana);

- consumo regular de doces (hábito de consumir doces em cinco ou mais dias por semana);

- fisicamente inativos (adultos que não praticaram qualquer atividade física no tempo livre nos últimos três meses, que não realizam esforços físicos intensos no trabalho, que não se deslocam para 0 trabalho ou escola a pé ou de bicicleta e que não são responsáveis pela "limpeza pesada" de suas casas);

- entrevistados que costumam assistir à televisão por 3 ou mais horas/dia;

- consumo abusivo de bebidas alcoólicas (hábito de consumir cinco ou mais doses para homens e quatro ou mais doses para mulheres em uma única ocasião nos últimos 30 dias);

- indivíduos que referiram conduzir veículo motorizado após consumo de qualquer quantidade de bebida alcoólica, independentemente da quantidade de bebida consumida e da frequência dessa situação;

- indivíduos que avaliaram seu estado de saúde como ruim;

- adultos que referiram diagnóstico médico de hipertensão arterial; e

- adultos que referiram diagnóstico médico de diabetes.

Os indicadores relacionados aos fatores de proteção foram avaliados segundo a frequência de:

- consumo de frutas e hortaliças regularmente (em cinco ou mais dias da semana);

- consumo recomendado de frutas e hortaliças (em cinco ou mais vezes por dia - equivalente a cinco porções/dia - em cinco ou mais dias da semana);

- consumo regular de feijão (em cinco ou mais dias da semana);

- prática do nível recomendado de atividade física no tempo livre (pelo menos 150 minutos semanais de atividade física de intensidade leve ou moderada, ou pelo menos 75 minutos semanais de atividade física de intensidade vigorosa, independentemente do número de dias em que pratica atividade física por semana);

- prática de atividade física no deslocamento (adultos que se deslocam para o trabalho ou escola de bicicleta ou caminhando e que despendem pelo menos 30 minutos diários no percurso de ida e volta);

- mulheres (de 50 a 69 anos de idade) que realizaram exame de mamografia nos últimos dois anos;

- mulheres (de 25 a 64 anos de idade) que realizaram exame de citologia oncótica (Papanicolau) para câncer de colo do útero nos últimos três anos.

Para o cálculo das prevalências, foi utilizado como denominador o total de adultos entrevistados, à exceção daqueles específicos a determinado sexo e faixa etária. Detalhes metodológicos podem ser encontrados em publicações anteriores. ${ }^{6,7}$

As análises dos dados foram realizadas por meio do aplicativo Stata 11.0. ${ }^{8}$ Foram utilizados fatores de ponderação do Vigitel que levam em consideração a probabilidade desigual que indivíduos residentes em domicílios com maior número de linhas telefônicas ou menor número de moradores tiveram em participar da amostra, além de corrigir a super ou subestimação da amostra do Vigitel decorrente da cobertura de telefonia fixa desigual no Brasil (utilizando-se procedimento de pós-estratificação). Assim, a ponderação envolveu três fatores: 0 inverso do número de linhas telefônicas no domicílio do entrevistado, o número de adultos no domicílio do entrevistado e o peso pós-estratificação, construído segundo as características de sexo, idade e escolaridade da amostra e da população total das capitais brasileiras (estimada a partir dos dados do censo demográfico de 2010 e interpolações intercensitárias). . Maiores informações sobre a construção dos fatores de ponderação utilizados pelo Vigitel podem ser encontrados no relatório anual da publicação. ${ }^{7}$

As estimativas das prevalências foram apresentadas em proporções (\%), com seus respectivos intervalos de confiança de $95 \%\left(\mathrm{IC}_{95 \%}\right)$. As prevalências foram estimadas segundo sexo (masculino e feminino), faixa etária $(18$ a 24, 25 a 34, 35 a 44, 45 a 54, 55 a 64 e 65 ou mais anos) e nível de escolaridade (zero a 8, 9 a 11 e 12 ou mais anos de estudo). Para analisar as diferenças nas prevalências segundo sexo, idade e escolaridade, foram estimadas as razões de prevalências (RP) ajustadas por idade, escolaridade ou ambos, e seus respectivos $\mathrm{IC}_{95 \%}$, por meio de modelo de regressão de Poisson com variância robusta, tendo a última categoria como referência. 
0 inquérito Vigitel foi aprovado pela Comissão Nacional de Ética em Pesquisa (CONEP), do Ministério da Saúde, sob o Parecer $\mathrm{n}^{0} 749 / 2006$ e registro $\mathrm{n}^{0}$ 13.081. A assinatura do Termo de Consentimento Livre e Esclarecido, nesse caso, foi substituída pelo consentimento verbal do entrevistado no momento da ligação telefônica. As ligações para todo o país foram feitas desde uma central telefônica e todas as entrevistas foram gravadas visando ao controle de qualidade dos dados. Não foram solicitados dados de identificação dos entrevistados, garantindo-se a confidencialidade e o sigilo das informações.

\section{Resultados}

Em 2012, foram entrevistados 45.448 adultos, com duração média da entrevista de 11 minutos, variando entre cinco e 58 minutos.

A prevalência de fumantes foi de $12,1 \%$, de fumantes passivos no domicilio foi de $10,2 \%$, de fumantes passivos no local de trabalho foi de $10,4 \%$ e a prevalência registrada para ex-fumantes foi de $21,1 \%$. Aproximadamente, metade da população apresentou excesso de peso $\left(51,0 \%\right.$; $\left.\mathrm{IC}_{95 \%}: 50,1 \%-51,9 \%\right)$, e $17,4 \%$, obesidade $\left(\mathrm{IC}_{95 \%}: 16,7 \%-18,1 \%\right)$. No que se refere aos hábitos alimentares, a prevalência do consumo de carne com excesso de gordura foi de $31,5 \%$, de consumo regular de refrigerantes ou suco artificial foi de $26,0 \%$, e do consumo de doces, de $18,8 \%$. Foram considerados fisicamente inativos $14,9 \%$ dos entrevistados e $26,4 \%$ relataram o hábito de assistir televisão 3 ou mais horas/ dia. 0 consumo abusivo de bebidas alcoólicas foi relatado por $18,4 \%\left(\mathrm{IC}_{95 \%}: 17,7 \%-19,2 \%\right)$ dos entrevistados e o hábito de ter dirigido um veículo após consumo de bebidas alcoólicas em qualquer quantidade, por 7,0\% (IC ${ }_{95 \%}:$ 6,6\%-7,5\%) (Tabela 1).

Em relação à prevalência dos fatores de proteção, o consumo regular e recomendado de frutas e hortaliças foi apresentado por $34,0 \%$ e $22,7 \%$ dos entrevistados, respectivamente, e o consumo regular de feijão, por $67,5 \%$. Os indivíduos que praticam 0 nível recomendado de atividade física no tempo livre representaram $33,5 \%$ ( $\left.\mathrm{IC}_{95 \%}: 32,6 \%-34,3 \%\right)$, e os que praticam atividade física no deslocamento, 14,2\% $\left(\mathrm{IC}_{95 \%}: 13,5-14,9\right)$ (Tabela 1).

Os diagnósticos médicos de hipertensão arterial e diabetes foram referidos por $24,3 \%$ e $7,4 \%$ da amostra, respectivamente. A prevalência registrada da realiza- ção de exames preventivos para detecção precoce de câncer foi de $77,4 \%\left(\mathrm{IC}_{95 \%}: 75,7 \%-79,2 \%\right)$ para mamografia nos últimos dois anos, em mulheres de 50 a 69 anos de idade; e de 82,3\% ( IC $\left._{95 \%}: 81,3 \%-83,3 \%\right)$ para exame de câncer de colo do útero (Papanicolau) nos últimos três anos, em mulheres de 25 a 64 anos (Tabela 1).

Entre os homens, identificou-se prevalência superior de: fumantes; ex-fumantes; fumantes com consumo de 20 ou mais cigarros por dia; fumo passivo no trabalho; consumo de refrigerante; consumo de carne com excesso de gordura; consumo de leite com gordura; e excesso de peso. Os homens ainda apresentam maior prevalência de consumo abusivo de bebidas alcoólicas, condução de veículo após consumo de álcool, atividade física no tempo livre, deslocamento ativo para o trabalho e inatividade física. Por outro lado, as mulheres apresentaram maiores prevalências de fumo passivo no domicílio, consumo de frutas e hortaliças (regular e recomendado), consumo de doces, avaliação de estado de saúde ruim, além de maior referência ao diagnóstico de hipertensão arterial (Tabela 1).

Observou-se uma diminuição do consumo de carne com excesso de gordura, consumo de refrigerantes e consumo regular de feijão à medida que aumentava a idade, embora 0 aumento da idade tenha-se associado positivamente ao consumo recomendado de frutas e hortaliças, ao excesso de peso/obesidade e ao diagnóstico de diabetes e hipertensão (Tabelas 2a e 2b).

0 consumo abusivo de álcool chegou a ser 4,6 vezes maior no grupo mais jovem ( 25 a 34 anos), quando comparado ao de indivíduos na faixa de idade mais elevada ( 65 ou mais anos). A prevalência de fumantes tendeu a aumentar até a faixa etária de 45 a 54 anos, reduzindo-se após essa idade. A inatividade física aumentou a partir dos 55 anos de idade, sendo maior entre os idosos (65 ou mais anos), enquanto a prática do nível recomendado de atividade física no tempo livre reduziu-se pela metade entre idosos. Os diagnósticos referidos de hipertensão arterial e diabetes foram mais frequentes entre os idosos (Tabelas 2a e 2b).

Quanto à escolaridade, o tabagismo apresentou maior frequência entre a população com até 8 anos de estudo, tendência semelhante àquela observada para a frequência de ex-fumantes, de fumo passivo no domicilio e no local de trabalho, de consumo de carnes com excesso de gordura, de consumo de refrigerante e de feijão, e do hábito de assistir televisão 
por 3 ou mais horas/dia. Por sua vez, os indivíduos de baixa escolaridade apresentaram menor frequência de consumo de frutas e hortaliças (Tabelas 3 e 4 ).

$\mathrm{Na}$ avaliação dos indicadores segundo sexo e escolaridade, destacam-se os indicadores de excesso de peso: para as mulheres, a prevalência foi maior entre aquelas com menor escolaridade; e para os homens, verificou-se situação contrária, com o excesso de peso apresentando-se menor em homens menos escolarizados (Tabelas $3 \mathrm{e} 4$ ).

Tanto em homens como em mulheres, a maior razão de prevalência - RP - para o consumo abusivo de bebidas alcoólicas e o hábito de dirigir após consumo de álcool foi verificada na população com mais escolaridade. A prática de atividade física no tempo livre também foi mais frequente entre homens e mulheres que estudaram por 12 ou mais anos, enquanto o hábito de assistir TV 3 horas ou mais por dia teve maior proporção na população com menos anos de estudo. Maior RP foi encontrada na população menos escolarizada para os diagnósticos referidos de hipertensão arterial e diabetes, bem como para a avaliação de saúde ruim, em ambos os sexos (Tabelas 3 e 4).

\section{Discussão}

No presente estudo, verificou-se que 0 excesso de peso atingiu mais da metade da população adulta brasileira e a obesidade quase um quinto, concomitantes à alimentação inadequada, marcada por baixo consumo de frutas e hortaliças e consumo excessivo de gorduras, além do consumo elevado de bebidas alcoólicas e açucaradas. A prática de atividade física foi observada em um terço da população estudada, apesar de importantes diferenças encontradas segundo idade e sexo. Mulheres e idosos praticaram menos atividade física; ademais, a população gastou longo tempo em frente à TV e deslocou-se para o trabalho de forma pouco ativa. 0 tabagismo prevaleceu na população masculina, com menor escolaridade e mais velha. As doenças autorreferidas, como diabetes e hipertensão arterial, predominaram entre mulheres e idosos. A maior prevalência dos fatores de risco em geral esteve associada ao sexo masculino e à baixa escolaridade.

0 excesso de peso e a obesidade constituem o sexto fator de risco mais importante para a carga global de doenças e estão associados com várias DCNT, como doenças cardiovasculares, diabetes, câncer de cólon, de reto e de mama, cirrose, entre outras. ${ }^{1,3,10-12}$ Estima- tivas de 2012, realizadas pela Federação Mundial de Obesidade, indicam que um bilhão de adultos esteja com sobrepeso no mundo e cerca de 475 milhões sejam obesos. ${ }^{11} 0$ crescimento do excesso de peso e da obesidade tem-se apresentado como contínuo, desde o início do monitoramento do Vigitel em 2006. ${ }^{6,7}$ Os dados do Vigitel estão coerentes com a transição nutricional e com os dados antropométricos aferidos na Pesquisa de Orçamentos Familiares (POF) de 2008. ${ }^{13}$

A associação entre menor escolaridade (proxy de nível socioeconômico) e excesso de peso e obesidade tem sido observada em outros estudos no Brasil. ${ }^{13-16}$ Este achado vem sendo explicado pelo menor poder aquisitivo das populações com menor escolaridade, consumo de alimentos de baixo custo e altamente energéticos, além de menor oportunidade para a prática de atividade física nessa população. ${ }^{13-15}$ A relação inversa entre excesso de peso e obesidade e o nível de escolaridade em mulheres foi descrita anteriormente, em estudos nacionais, ${ }^{13,15,16} 0$ que pode ser justificado pela maior preocupação com a magreza e estética entre as mulheres, enquanto entre os homens não existe esse padrão cultural. ${ }^{16}$ Essas explicações ainda precisam ser melhor compreendidas, inclusive mediante estudos qualitativos.

Edições anteriores do Vigitel mostraram prevalências menores de diabetes. ${ }^{6} \mathrm{O}$ aumento da prevalência do diabetes tem sido descrito em outros países do mundo, associado ao aumento da prevalência da obesidade. ${ }^{17} 0$ uso da morbidade referida de diabetes e hipertensão vem sendo descrito como fator para subestimar as prevalências. Outros estudos mostram que essa metodologia tem se mostrado útil e válida, ${ }^{17-20}$ além de esses dados serem mais fáceis de obter e a menor custo. ${ }^{21}$ No Brasil, maiores prevalências em mulheres têm sido descritas quando se utiliza dados autorreferidos, pelo fato de elas frequentarem mais os serviços de saúde e terem maior oportunidade de diagnóstico, ${ }^{22}$ não obstante 0 presente estudo ter encontrado diferenças significativas apenas para a prevalência de hipertensão arterial.

A prevalência de fumantes no Brasil era de 34,8\% no final da década de 1980 e apresentou declínio consistente ao longo dos anos. ${ }^{23,24} \mathrm{O}$ Brasil tem sido considerado uma liderança global na implementação da Convenção-Quadro para o Controle do Tabaco e apresenta a menor prevalência no estudo comparado do Global Adult Tobbaco Survey (GATS) ${ }^{25} 0$ Vigitel 


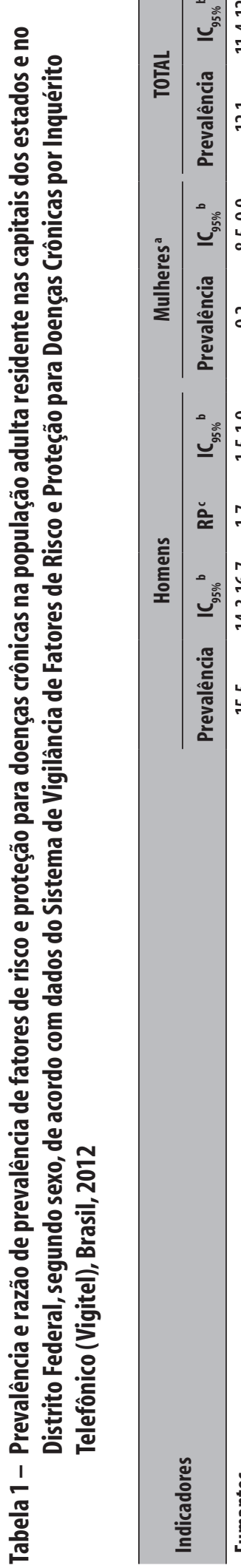

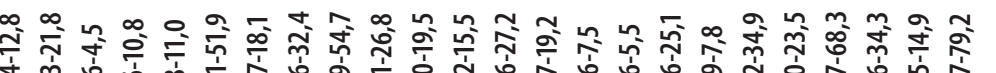

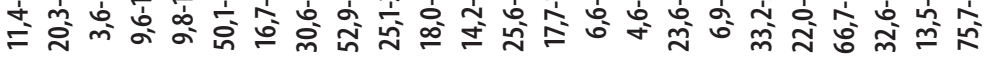

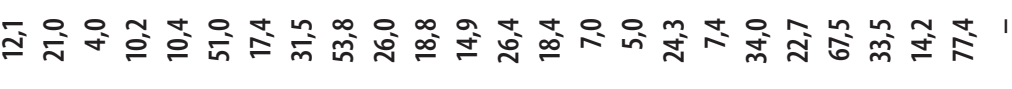

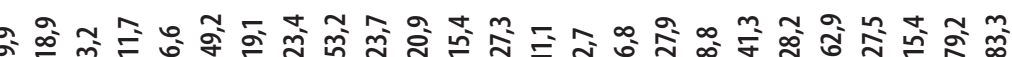

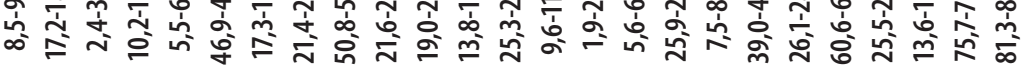

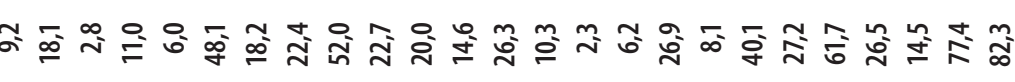

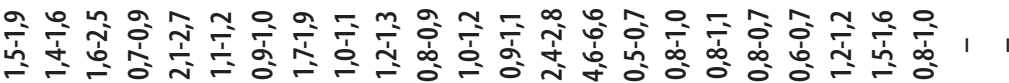

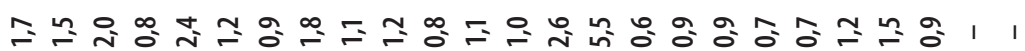

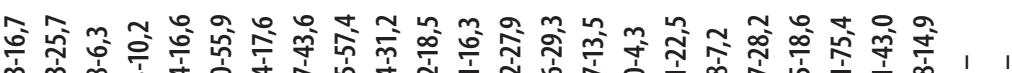

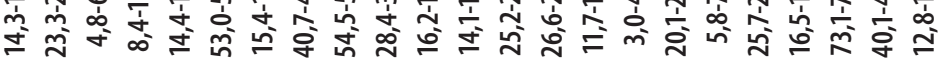

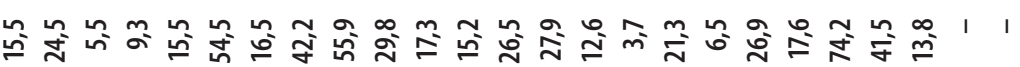

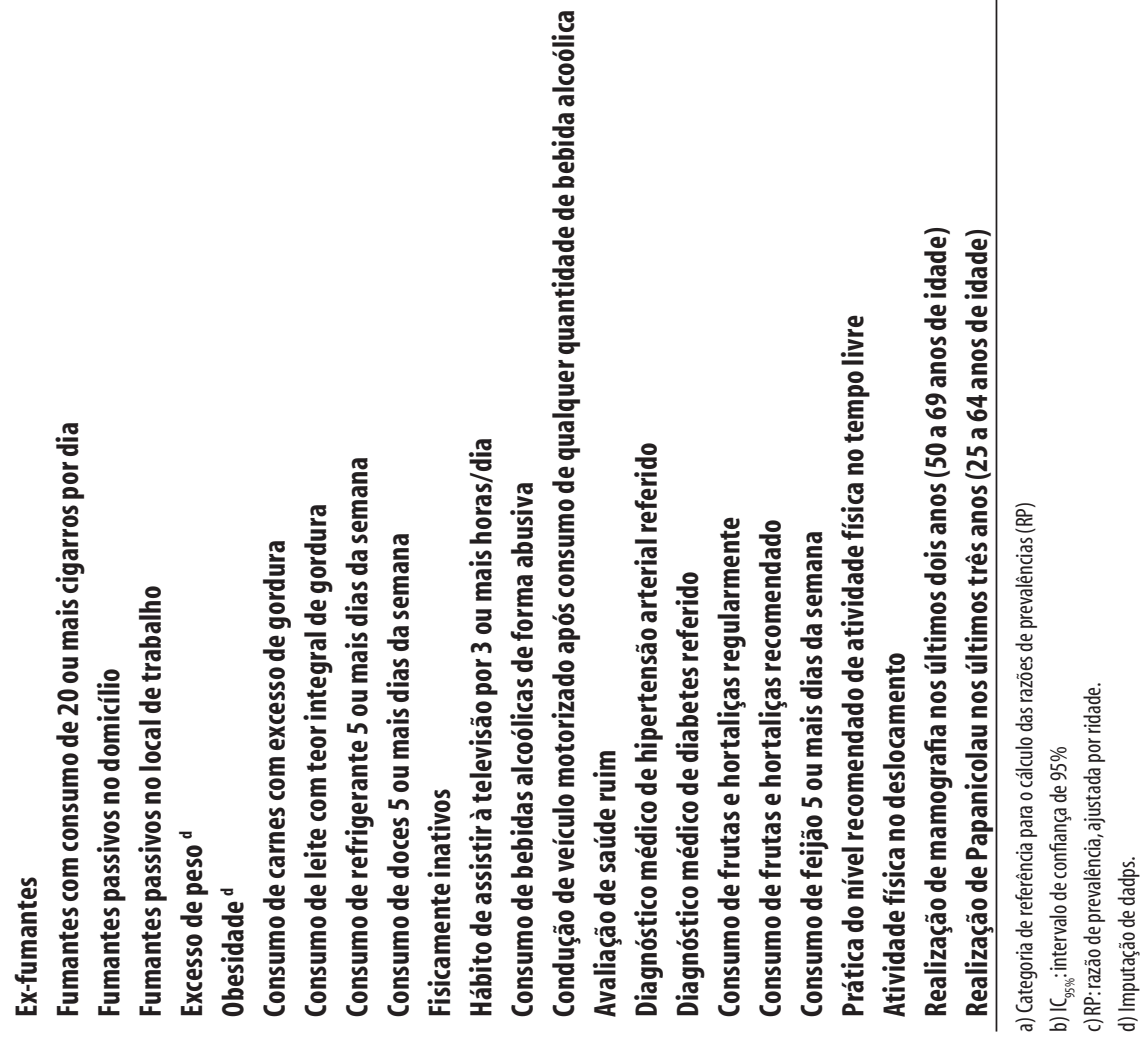




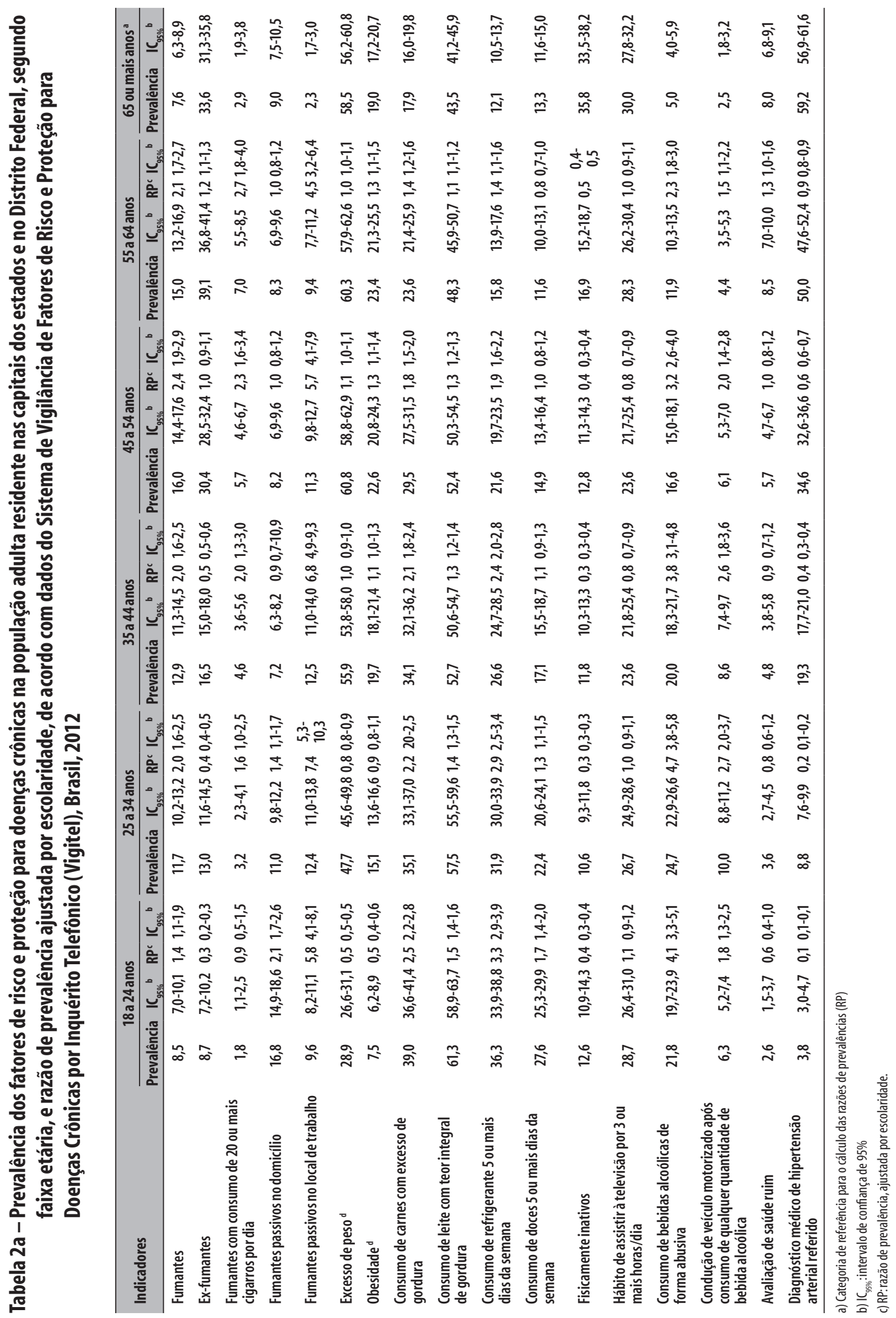




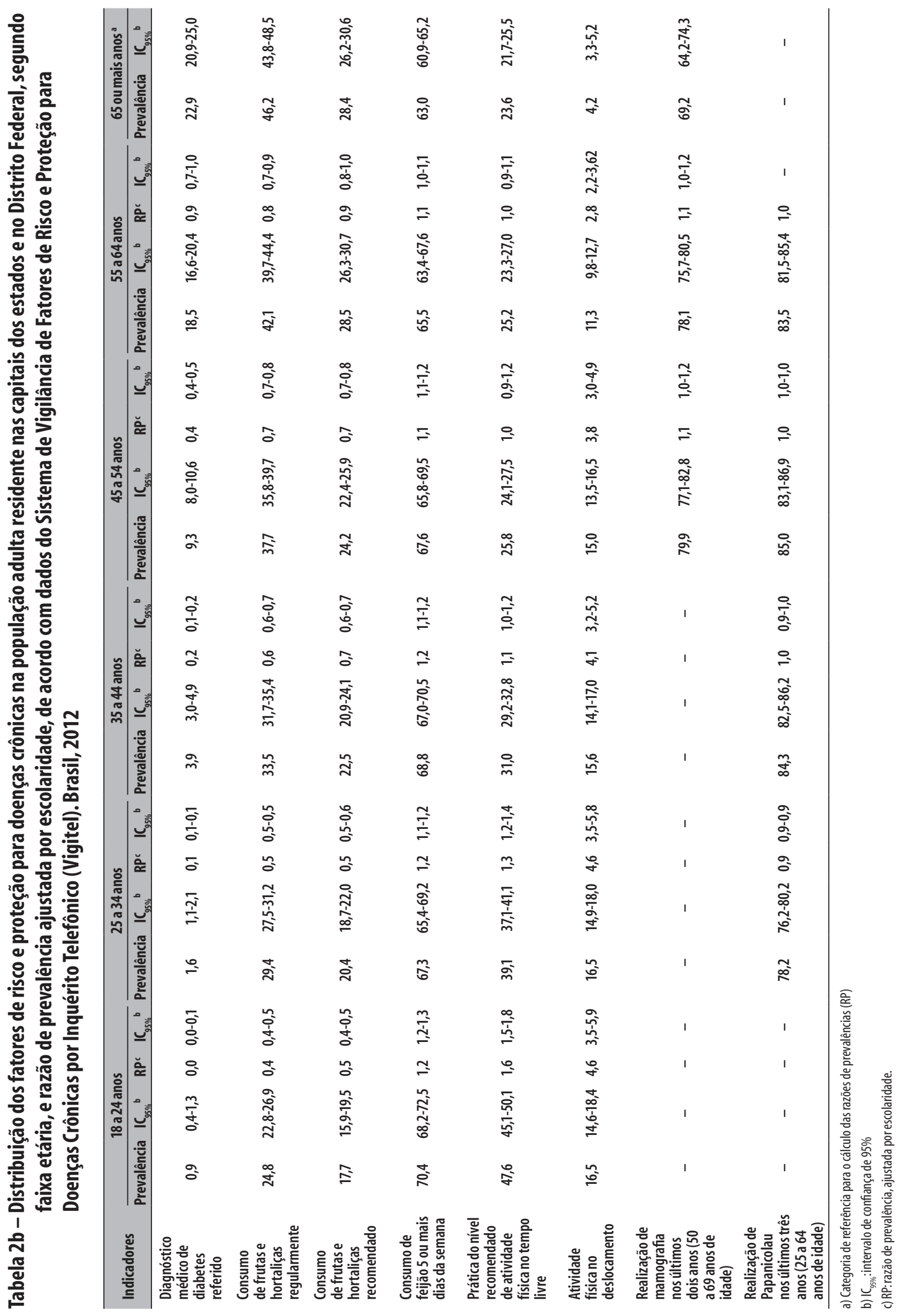




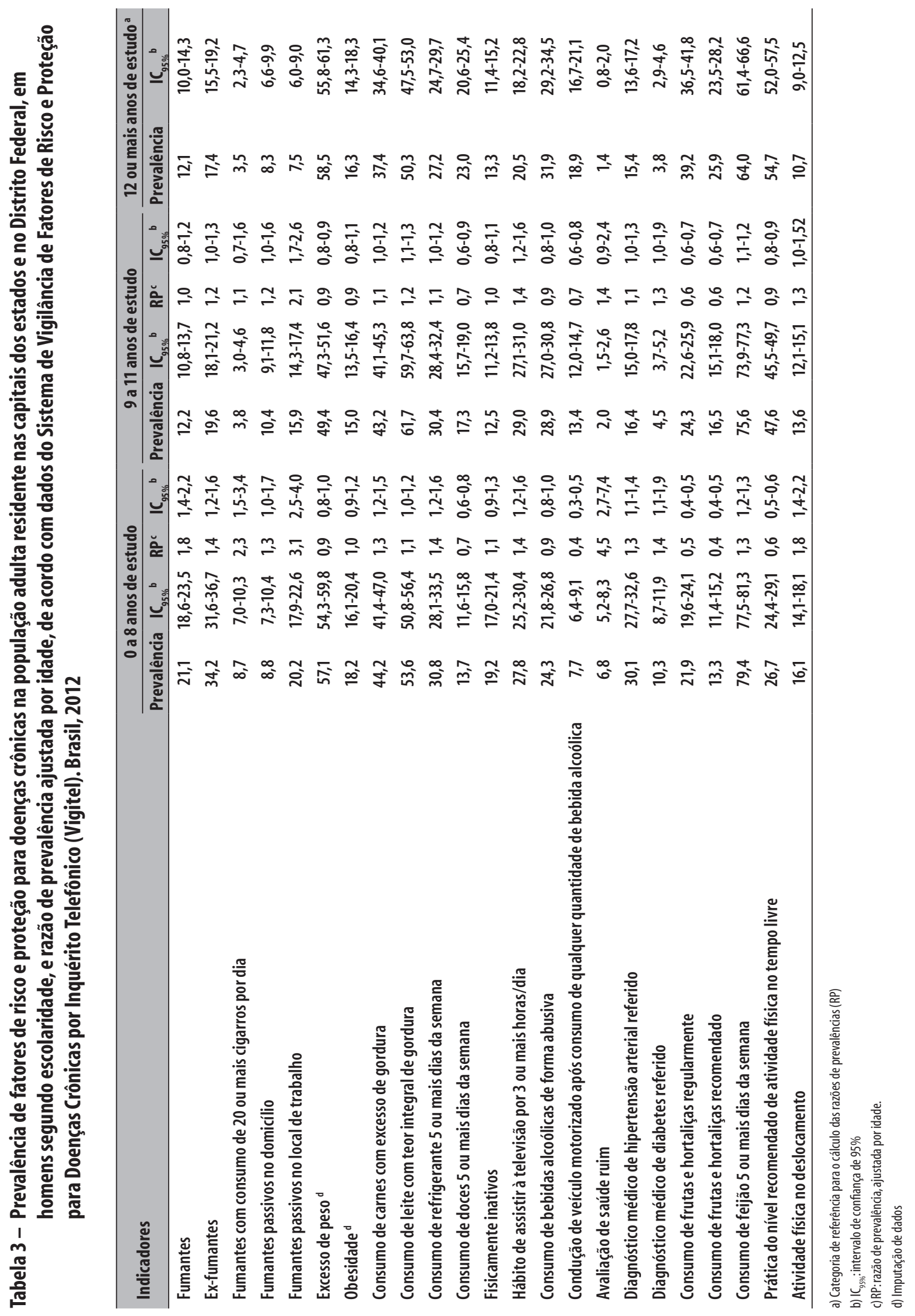




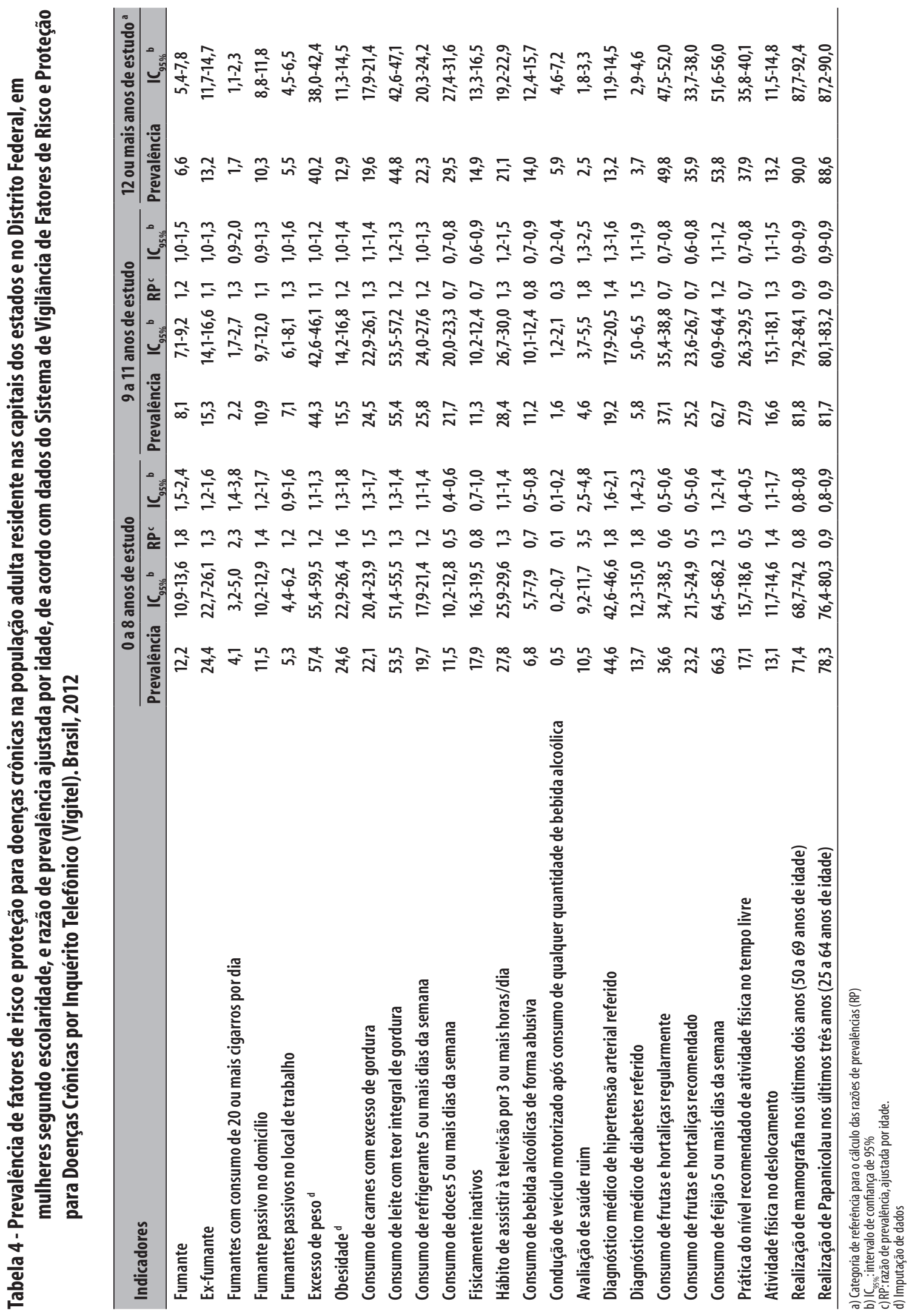


2012, pela primeira vez, apontou a redução do tabagismo também em mulheres, além de mostrar prevalência mais elevada de fumantes na população com menor escolaridade e na população acima de 40 anos de idade, declinando em idosos. 0 fumo passivo, tanto em casa quanto no trabalho, foi maior na população com menor escolaridade, o que também foi demonstrado pela Pesquisa Nacional por Amostra de Domicílios (PNAD) realizada em 2008. ${ }^{26} 0$ fumo passivo em casa foi maior entre as mulheres, podendo ser explicado por seu maior tempo de exposição no domicílio, além da maior prevalência de tabagismo entre homens. ${ }^{26}$ Em 2011, foi promulgada nova lei que proíbe fumar em locais públicos, além do aumento das alíquotas dos impostos de cigarros, levando a perspectivas muito promissoras de alcance da meta de redução do tabagismo em 30\% antes de 2022, prevista no Plano de Ações Estratégicas para o Enfrentamento das DCNT., ${ }^{54}$

O consumo de álcool está associado a diversas doenças crônicas, incluindo doenças do fígado, cânceres, doenças mentais, além de agravos como acidentes. A Organização Mundial da Saúde (OMS) adotou uma meta de redução relativa de $10 \%$ no consumo per capita de álcool entre a população $\geq 15$ anos até $2025,5,27$ a partir de medidas para torná-lo mais caro e assim, menos disponível. No Brasil, o consumo abusivo de bebidas alcoólicas, medido pelo Vigitel, mostra-se mais frequente em homens, mais jovens e de maior escolaridade. Iniciativas específicas para essa população devem ser implementadas, entre as quais se incluem ações regulatórias para o comércio, publicidade e propaganda, bem como o aumento da fiscalização no setor, medidas previstas no Plano de Ações Estratégicas para o Enfrentamento das DCNT,,

A atividade física insuficiente constitui a quarta principal causa de morte no mundo. ${ }^{3}$ Existem importantes diferenciais quanto a sexo, escolaridade e idade em relação aos indicadores de atividade física. 0 Vigitel indicou que a atividade física no lazer está associada a maior escolaridade, sexo masculino e idade jovem, conforme apontado em outros estudos. ${ }^{28} \mathrm{~A}$ atividade física do deslocamento está mais associada ao sexo masculino e à baixa escolaridade, enquanto a inatividade física é mais presente em idosos, conforme descrito na literatura. ${ }^{28}$

A maior frequência de fatores de risco como tabagismo, excesso de peso, hipertensão arterial e diabetes entre adultos menos escolarizados (especialmente mu- lheres) expressa as desigualdades sociais existentes no país, ${ }^{26,29}$ enfatizando o papel do Sistema Único de Saúde (SUS) no sentido de buscar a equidade do cuidado em saúde, ao fornecer acesso aos serviços de saúde com tratamento adequado para toda a população. ${ }^{26}$

0 maior acometimento das doenças crônicas com 0 avanço da idade é um resultado esperado, pela característica dessas doenças. Aliado à ocorrência simultânea de diferentes fatores de risco e às mudanças corporais e funcionais inerentes ao processo de envelhecimento, esse resultado encontrado aponta para a necessidade de um cuidado especial voltado a essa fase da vida.

Os exames de mamografia e Papanicolau indicaram cobertura adequada na média, inclusive apontando que as metas do Plano de DCNT para a cobertura desses exames, de $70 \%$ e $85 \%$ respectivamente, já teriam sido atingidas. ${ }^{5}$ Os dados da PNAD $2008^{26}$ mostram maiores prevalências de realização desses exames entre mutheres de maior renda e escolaridade, diferenças que têm sido progressivamente reduzidas.

Entre as limitações inerentes à própria metodologia do inquérito telefônico Vigitel, existe uma restrição da amostra aos indivíduos residentes nas capitais de estados brasileiros e no Distrito Federal que possuem telefone fixo, limitando sua representatividade. Este problema é minimizado pelo uso de fatores de ponderação dos dados, os quais buscam estimar as prevalências levando em consideração as diferenças nas características demográficas da amostra do Vigitel em relação às características da população total, por meio da pós-estratificação. Em 2012, a pós-estratificação foi atualizada pelas projeções intercensitárias, a partir da liberação dos microdados do censo demográfico de 2010. Além disso, a cobertura de telefonia fixa no país vem sendo monitorada constantemente, pela equipe gestora do Vigitel.

O Plano de Enfrentamento das DCNT ${ }^{3,4}$ prioriza a redução da exposição da população aos fatores de risco e o incentivo aos fatores protetores, visando ampliar medidas de proteção da saúde, seja na criação de espaços para a prática de atividade física - a exemplo do Programa Academia da Saúde,$-{ }^{30}$ seja com medidas de proibição à propaganda do cigarro e criação de ambientes livres de fumo, entre outras, além do apoio a estilos de vidas saudáveis, pela melhoria da qualidade de vida e bem-estar da população.

0 estudo aponta os avanços já alcançados com a redução do tabagismo, e reforça a preocupação com 
a obesidade e o excesso de peso. Devem ser mantidos os esforços direcionados para o estímulo à prática de atividade física e de uma alimentação saudável. É mister considerar a diversidade de padrões de exposição a fatores de risco e proteção segundo sexo, idade e escolaridade, para serem contemplados nas estratégias de promoção da saúde.

\section{Contribuição dos autores}

Todos os autores participaram da concepção do estudo, análise e interpretação dos dados, redação, revisão e aprovação final do artigo, e são responsáveis por todos os seus aspectos, incluindo a garantia de sua precisão e integridade.

\section{Grupo de elaboração do Vigitel 2012}

Ana Paula Borloletto Martins

Núcleo de Pesquisas Epidemiológicas em Nutrição e Saúde, Universidade de São Paulo, São Paulo-SP, Brasil

Cristiane Penaforte do Nascimento Dimech

Secretaria de Saúde, Prefeitura do Recife-PE, Brasil

Secretaria de Vigilância em Saúde, Ministério da Saúde, Brasília-DF, Brasil

Daniela Silva Canella

Núcleo de Pesquisas Epidemiológicas em Nutrição e Saúde, Universidade de São Paulo, São Paulo-SP, Brasil

\section{José Nilson dos Santos Júnior}

Expertise Inteligência e Pesquisa de Mercado, Belo Horizonte-MG, Brasil

\section{Juliano Ribeiro Moreira}

Expertise Inteligência e Pesquisa de Mercado, Belo Horizonte-MG, Brasi

Larissa Galastri Baraldi

Núcleo de Pesquisas Epidemiológicas em Nutrição e Saúde, Universidade de São Paulo, São Paulo-SP, Brasil

Lenildo de Moura

Organização Pan-Americana da Saúde, Brasília-DF, Brasil

Maria Aline Siqueira Santos

Secretaria de Vigilância em Saúde, Ministério da Saúde, Brasília-DF, Brasil

Maria Laura da Costa Louzada

Núcleo de Pesquisas Epidemiológicas em Nutrição e Saúde, Universidade de São Paulo, São Paulo-SP, Brasil

Marta Alves e Silva

Secretaria de Vigilância em Saúde, Ministério da Saúde, Brasília-DF, Brasil

Maryane Oliveira Campos

Secretaria de Vigilância em Saúde, Ministério da Saúde, Brasília-DF, Brasil

Micheline Gomes Campos da Luz

Secretaria de Vigilância em Saúde, Ministério da Saúde, Brasília-DF, Brasil

Naiane de Brito Francischetto

Secretaria de Vigilância em Saúde, Ministério da Saúde, Brasília-DF, Brasil

Paula Carvalho de Freitas

Secretaria de Vigilância em Saúde, Ministério da Saúde, Brasília-DF, Brasil

Regina Rodrigues

Núcleo de Pesquisas Epidemiológicas em Nutrição e Saúde, Universidade de São Paulo, São Paulo-SP, Brasil

Renan Kendy Mancio

Expertise Inteligência e Pesquisa de Mercado, Belo Horizonte-MG, Brasil

Taís Porto

Secretaria de Vigilância em Saúde, Ministério da Saúde, Brasília-DF, Brasil 


\section{Referências}

1. World Health Organization. WHO Global status report on noncommunicable diseases 2010 [Internet]. Geneva: World Health Organization; 2011 [cited 2012 Jun 17]. p. 176. Available from: http://www.who.int/ $\mathrm{nmh} /$ publications/ncd_report2010/en/

2. United Nations. Political declaration of the high-level meeting of the general assembly on the prevention and control of non-communicable diseases. In: High-level Plenary Meeting of the General Assembly [Internet]. 2011 Sep 19-20; New York: United Nations; 2011 [cited 2012 Jun 19]. Available from: http://www.un.org/ga/search/view_doc. asp?symbol=A/66/L.1

3. Ministério da Saúde (BR). Secretaria de Vigilância em Saúde. Departamento de Análise de Situação de Saúde. Plano de ações estratégicas para o enfrentamento das Doenças Crônicas Não Transmissíveis (DCNT) no Brasil 20112022 [Internet]. Brasília: Ministério da Saúde; 2011 [citado 2012 jun 17]. Disponível em: http://bvsms.saude.gov.br/bvs/publicacoes/ enfrentamento_doencas_cronicas_nao_ transmissiveis_2011_2022.pdf

4. Malta DC, Morais Neto OL, Silva Junior JB. Apresentação do plano de ações estratégicas para o enfrentamento das doenças crônicas não transmissíveis no Brasil, 2011 a 2022. Epidemiol Serv Saude. 2011 dez;20(4):425-38.

5. Malta DC, Silva Junior JB. O Plano de ações estratégicas para o enfrentamento das doenças crônicas não transmissíveis no Brasil e a definição das metas globais para o enfrentamento dessas doenças até 2025: uma revisão. Epidemiol Serv Saude. 2013 mar;22(1):151-64.

6. Ministério da Saúde (BR). Secretaria de Vigilância em Saúde. Secretaria de Gestão Estratégica Participativa. Vigitel 2006: vigilância de fatores de risco e proteção para doenças crônicas por inquérito telefônico. Brasília: Ministério da Saúde; 2007.

7. Ministério da Saúde (BR). Secretaria de Vigilância em Saúde. Departamento de Vigilância de Doenças e Agravos Não Transmissíveis e Promoção da Saúde. Vigitel 2012: vigilância de fatores de risco e proteção para doenças crônicas por inquérito telefônico. Brasília: Ministério da Saúde; 2013.
8. StataCorp. Stata Statistical Software: release 11. College Station, TX: StataCorp LP; 2009.

9. Izrael D, Hoaglin DC, Battaglia MP. A SAS macro for balancing a weighted sample. In: Proceedings of the Twenty-Fifth Annual SAS Users Group International Conference; 2000 Apr 9-12, Paper 275, Cary (NC): SAS Institute; 2000.

10. World Health Organization. Obesity: preventing and managing the global epidemic: reporter of WHO consultation. Geneva: World Health Organization; 2000. (WHO Technical Reporter Series; no. 894).

11. International Association for the Study of Obesity. Adult overweight and obesity in the European Union (EU27) [Internet]. London: International Association for the Study of Obesity; 2013 [cited 2013 Nov 3]. Available from: http://www.iaso.org/resources/worldmap-obesity/

12. Institute for Health Metrics and Evaluation. Global burden of diseases, injuries, and risk factors study 2010: Brazil [Internet]. Seattle: Institute for Health Metrics and Evaluation; 2010 [cited 2014 Oct 14]. Available from: http://www.healthdata.org/sites/ default/files/files/country_profiles/GBD/ihme_gbd_ country_report_brazil.pdf

13. Instituto Brasileiro de Geografia e Estatística. Pesquisa de orçamentos familiares 2008-2009: análise do consumo alimentar pessoal no Brasil. Rio de Janeiro: Instituto Brasileiro de Geografia e Estatística; 2011.

14. Monteiro CA, Conde WL, Castro IRR. The changing relationship between education and risk of obesity in Brazil (1975-1997). Cad Saude Publica. 2003;19 Suppl 1:S67-75.

15. Monteiro CA, Conde WL, Popkin BM. Independent effects of income and education on the risk of obesity in the Brazilian adult population. J Nutr. 2001 Mar;131(3):881S-6S.

16. Sichieri R, Moura EC. Variações no índice de massa corporal entre as capitais brasileiras: análise multinível. Rev Saude Publica. 2009 nov;43 supl 2:907.

17. Danaei G, Finucane MM, Lu Y, Singh GM, Cowan MJ, Paciorek CJ, et al. National, regional, and global trends in fasting plasma glucose and diabetes prevalence since 1980: systematic analysis of health 
examination surveys and epidemiological studies with 370 country-years and 2.7 million participants. Lancet. $2011 \mathrm{Jul} ; 378(9785): 31-40$.

18. Martin LM, Leff M, Calonge N, Garrett C, Nelson DE. Validation of self-reported chronic conditions and health services in a managed care population. Am J Prev Med. 2000 Apr;18(3):215-8.

19. Okura Y, Urban LH, Mahoney DW, Jacobsen SJ, Rodeheffer RJ. Agreement between self-report questionnaires and medical record data was substantial for diabetes, hypertension, myocardial infarction and stroke but not for heart failure. J Clin Epidemiol. 2004 Oct;57(10):1096-103.

20. Schmidt MI, Duncan BB, Hoffmann JF, Moura L, Malta DC, Carvalho RM. Prevalence of diabetes and hypertension based on self-reported morbidity survey, Brazil, 2006. Rev Saude Publica. 2009 Nov; 43 Suppl 2:74-82.

21. Mokdad AH, Remington PL. Measuring health behaviors in populations. Prev Chronic Dis. 2010 Jul;7(4):A75.

22. Lima-Costa MF, Peixoto SV, Firmo JO. Validade da hipertensão arterial auto-referida e seus determinantes (projeto Bambuí). Rev Saude Publica. 2004 out;38(5):637-42.

23. Ministério da Saúde (BR). Instituto Nacional de Câncer, Organização Pan-Americana da Saúde. Pesquisa especial de tabagismo - PETab: relatório Brasil. Rio de Janeiro: Instituto Nacional de Câncer; 2011.

24. Malta DC, Iser BPM, Sá NNB, Yokota RTC, Moura L, Claro RM, et al. Tendências temporais no consumo de tabaco nas capitais brasileiras, segundo dados do Vigitel, 2006 a 2011. Cad Saude Publica. 2013 abr;29(4):812-22.
25. Giovino GA, Mirza SA, Samet JM, Gupta PC, Jarvis MJ, Bhala $\mathrm{N}$, et al. Tobacco use in 3 billion individuals from 16 countries: an analysis of nationally representative cross-sectional household surveys. Lancet. 2012 Aug;380(9842):668-79.

26. Instituto Brasileiro de Geografia e Estatística. Pesquisa Nacional por Amostra de Domicílios: um panorama da saúde no Brasil: acesso e utilização dos serviços, condições de saúde e fatores de risco e proteção à saúde, 2008. Rio de Janeiro: Instituto Brasileiro de Geografia e Estatística; 2010.

27. World Health Organization. A comprehensive global monitoring framework, including indicators, and a set of voluntary global targets for the Prevention and Control of Noncommunicabale Diseases [Internet]. Geneva: World Health Organization; 2012 [cited 2012 Sep 6]. Available from: http://www.who.int/nmh/ events/2012/ncd_discussion_paper/en/index.html

28. Lee I-M, Shiroma EJ, Lobelo F, Puska P, Blair SN, Katzmarzyk PT, et al. Effect of physical inactivity on major non-communicable diseases worldwide: an analysis of burden of disease and life expectancy. Lancet. 2012 Jul;380(9838):219-29.

29. Barros MBA, Galvão CL, Carandina L, Torre GD. Social inequalities in the prevalence of chronic diseases in Brazil, PNAD-2003. Cienc Saude Coletiva. 2006 Dec;11(4):911-26.

30. Malta DC, Silva Junior JB. Policies to promote physical activity in Brazil. Lancet. 2012 Jul;380(9838):195-6.

Recebido em 14/03/2014

Aprovado em 15/10/2014 